\title{
Sozialwissenschaftliche Umfrageforschung im Umbruch? Datenqualität und Effizienz
}

\author{
Sylvia Kritzinger ${ }^{1, *}$, Katharina Götsch ${ }^{1,{ }^{* *}}$ \\ 1 Universität Wien, Institut für Staatswissenschaft, Austria \\ * E-Mail: sylvia.kritzinger@univie.ac.at \\ ** E-Mail: katharina.goetsch@univie.ac.at
}

Repräsentative Bevölkerungsumfragen werden in Österreich regelmäßig von verschiedenen Institutionen $\mathrm{zu}$ unterschiedlichen Zwecken konzipiert und durchgeführt. Private Meinungsforschungsinstitute, sozial- und wirtschaftswissenschaftliche Forschung an den Universitäten und außeruniversitären Forschungseinrichtungen sowie nicht zuletzt die Statistik Austria beschäftigen sich aus verschiedenen Perspektiven mit Personenbefragungen. Die Themenpalette, die Umfragen dabei umfassen, ist enorm: sie reicht von Daten zur Soziodemografie der BürgerInnen bis zur Erfassung ihrer politischen, sozialen, wirtschaftlichen Einstellungen und Meinungen sowie Verhaltensweisen.

In der empirischen Sozialforschung sind Daten aus Umfragen eine gewichtige „Währung“ in der Beantwortung zentraler Forschungsfragen. Aber nicht nur die Wissenschaft, auch die Gesellschaft allgemein, Politik, Medien etc. brauchen qualitativ hochwertige Informationen über die Bevölkerung als solide Grundlage für das Treffen von Entscheidungen, das Einordnen von Veränderungen oder als Orientierung in der Gesellschaft. Umso wichtiger ist es, dass Umfragedaten von exzellenter Qualität sind, die Bevölkerung repräsentativ abbilden und somit erlauben, Rückschlüsse auf die Bevölkerung zu ziehen.

Die Umfrageforschung befindet sich aktuell durchaus in einer Phase des Umbruchs und der Neuorientierung. Einerseits eröffnen technologische Entwicklungen wie Smart Phones oder Tablets (,mobile devices') vielfältige Möglichkeiten der Durchführung von Umfragen, die bereits durch die Möglichkeit von Onlineumfragen Mitte der Nullerjahre stark verändert wurden. Kostengünstigere Umfragen, schnellere Erreichbarkeit der RespondentInnen, Blitzumfragen nach TV-Ereignissen und somit beinahe Echtzeit-Daten erlauben es, empirische Informationen schneller und effizienter zu sammeln. Andererseits können diese Neuerungen auch Qualitätsverluste mich sich bringen, da bestimmte Modi (dies sind Erhebungen via Telefon, Face-to-face, Online, Papier etc.) oder Geräte (Handy, Tablet etc.) Personengruppen von der Teilnahme an Umfragen ausschließen können. Dies gilt etwa für ältere Personen, Haushalte ohne Internetzugang usw.

Aspekte der Erreichbarkeit von RespondentInnen sowie die methodologischen Folgen der Umstellung auf neue Geräte und Modi gilt es zu berücksichtigen und zu bewerten, um dem Anspruch repräsentativer Umfragen, die Gesamtbevölkerung abbilden zu können, gerecht zu werden. Besonders im Fokus stehen dabei Umfragen bei Wahlen zu Landtagen und zum Nationalrat, wenn Vorhersagen aus Umfragen vom tatsächlichen Wahlergebnis mehr oder weniger stark abweichen. Allgemein birgt die steigende gesellschaftliche, politische und mediale Nachfrage nach empirischen Daten über Einstellungen, Meinungen und Verhalten von BürgerInnen auch Risiken eines Qualitätsverlusts: der Zugang zu Daten per se in Abwägung zur Qualität der Daten.

Dies sind nur einige Aspekte, auf die sich Institutionen im Bereich der empirischen Sozialforschung einstellen müssen. Das vorliegende Schwerpunktheft der Österreichischen Zeitschrift für Politikwissenschaft setzt bei dieser Problematik an und greift einige dieser Fragen auf. Die im Schwerpunktheft publizierten Beiträge stützen sich auf eine Ende 2016 stattgefundene Konferenz der Plattform für Umfragen, Methoden und empirische Analysen (PUMA) unter dem Titel „Umfrageforschung in Österreich: Vernetzung - Austausch - Weiterentwicklung“. Rund 20 Vorträge aus der universitären und außeruni- 
versitären Methodenforschung, der privatwirtschaftlichen Meinungsforschung sowie der amtlichen Statistik beschäftigten sich dabei mit neuen Herausforderungen in quantitativen Umfragen in Österreich und daraus resultierenden Qualitätsaspekten. Für das vorliegende Heft der ÖZP hat PUMA (vertreten durch die PUMALeiterin Univ.-Prof. Dr. Sylvia Kritzinger und Projektkoordinatorin Dr. Katharina Götsch) aus den gehaltenen Vorträgen eine Auswahl an innovativen, kritischen und für den Bereich der Umfrageforschung relevanten Themen getroffen, die für dieses Schwerpunktheft noch weiter ausgearbeitet und in einem Begutachtungsverfahren evaluiert wurden.

Die übergreifende Fragestellung des Schwerpunktheftes widmet sich folgenden Aspekten: Wie können technische, gesellschaftliche und methodologische Innovationen und Trends für die Weiterentwicklung der Umfrageforschung genutzt werden, um die Datenqualität zu sichern und die Effizienz zu optimieren? Dieser Frage gehen die im Folgenden kurz dargestellten Beiträge aus verschiedenen Blickwinkeln nach.

Die ersten Beiträge des Schwerpunkthefts fokussieren auf Qualitätsaspekte im Datenerhebungsprozess, wobei zunächst Fragen der Repräsentativität in Onlinebefragungen nachgegangen wird und anschließend Effekte von InterviewerInnen betrachtet werden.

Alexander Seymer und Martin Weichbold berichten in ihrem Artikel „Social Inequalities and the Effects of Incentives on Web Survey Participation" von Rekrutierungsexperimenten für eine Onlinebefragung der PUMA-Plattform im Sommer 20I6. Die von Statistik Austria durchgeführte Erhebung testete verschiedene Incentivevarianten im Hinblick auf Teilnahmeraten und Stichprobenverzerrungen. Dabei konnte nachgewiesen werden, dass sowohl der sozioökonomische Status als auch der Bildungsgrad von RespondentInnen eine bedeutende Rolle bei der Rücklaufquote sowie der Präferenz für bestimmte Incentivearten spielen. Die Ergebnisse bieten eine empirische Grundlage für optimierte Rekrutierungsstrategien.

Anschließend geht Julian Aichholzer in seinem Beitrag anhand von Daten zur Nationalratswahl 2013 - erhoben von der Austrian National Election Study (AUTNES) - der Frage nach, wie und warum verschiedene Befragungsmodi zu ähnlichen Ergebnissen führen können. Angesichts der Tatsache, dass RespondentInnen mittels unterschiedlicher Modi besser erreicht und befragt werden können - und somit die Ausschöpfungsquote erhöht werden kann -, gibt dieser Beitrag wichtige Einblicke, wie mit zukünftigen Daten aus Multi-Mode-Verfahren umgegangen werden kann. Am Beispiel von FPÖ-WählerInnen weist Aichholzer nach, dass der Web-Modus im Vergleich zu klassischen Modi wie z.B. Face-to-FaceBefragungen zum einen eher politisch unzufriedene und entfremdete RespondentInnen erreicht; zum anderen stellt er fest, dass Effekte der sozialen Erwünschtheit in anonymen Webbefragungen deutlich geringer sind als bei persönlichen Befragungen.

Ebenfalls mit Onlinebefragungen beschäftigt sich der gemeinsame Beitrag von Monika Mühlböck, Nadia Steiber und Bernhard Kittel. Basierend auf einer Panelstudie unter jungen Arbeitslosen zwischen 18 und 28 Jahren in Österreich werden Effekte der InterviewerInnenpräsenz bzw. -absenz untersucht. Empfehlungen aus früherer Forschungsliteratur zur Steigerung der Teilnahme schwer erreichbarer Zielgruppen (Jugendliche, Personen mit sprachlichen Schwierigkeiten, Migrationshintergrund, niedrigem Bildungsgrad, etc.) wurden im Rahmen der Studie in der Praxis getestet. Deren Ergebnisse heben etwa die Bedeutung einfacher Frageformulierungen, die Kombination von Face-to-Face-Rekrutierung und Computer-gestützten Selbstausfüllerfragebogen, Incentives, InterviewerInnenverhalten sowie eine hohe Anzahl an Kontaktierungen als Möglichkeiten hervor, die Teilnahmebereitschaft bei jungen RespondentInnen zu erhöhen.

InterviewerInnen und deren Einflussfaktoren stehen auch im Zentrum des Beitrags von Nicole Halmdienst und Michael Radhuber, die das Qualitätsmanagement der europäischen Umfrage SHARE darstellen und kritisch diskutieren. Die AutorInnen betonen die Bedeutung eines fortlaufenden Monitorings und Qualitätsmanagements im Sinne einer aktiven Begleitung der InterviewerInnen während der Feldphase. Als Ergebnis ihrer Analyse schlagen Halmdienst und Radhuber harmonisierte Standards für die Implementierung hochqualitativer Umfragen vor wie etwa detaillierte Protokolle der InterviewerInnen über Kontaktierungen und Rückläufe oder auch externe Überprüfungen. Darüber hinaus werden Empfehlungen zum Sampling und zur Zusammenarbeit mit externen Datenerhebungsinstituten als Schlussfolgerungen der SHARE-Erfahrungen formuliert.

Abschließend fragen Johann Bacher und Dimitri Prandner nach den Möglichkeiten und dem potenziellen Nutzen der Fusion verschiedener Datensätze für die empirische Sozialforschung und stellen die relevanten Ergebnisse eines solchen Datenfusionsexperiments für den Sozialen Survey Österreichs (SSÖ) 2016 und den European Social Survey (ESS) 2015 dar. Die Autoren prüfen die Qualität von Datenfusionen anhand der Fragestellung, welchen Kandidaten der Bundespräsidentschaftswahl 2016 die WählerInnen der Nationalratswahl 2013 präferierten. Die Verbindung zweier unterschiedlicher Datensätze kann dabei sowohl zu erhöhter als auch zu reduzierter Qualität der neu generierten Daten führen. Eine qualitativ hochwertige Fusion mittels Standardstatistikprogrammen ist durchaus möglich, während ein allgemeines Fusionsmodell nicht überzeugen konnte.

Im Allgemeinen zeigen die Beiträge dieses Schwerpunkthefts auf, dass im Bereich der Umfrageforschung 
ein sich lohnendes Forschungsfeld liegt, deren Erkenntnisse für weiterführende sozialwissenschaftliche Forschungsfragen von großer Bedeutung sind (sofern auf quantitativen Daten basierend), die mit einbezogen werden sollen, um inhaltlich richtige Schlüsse zu ziehen. Methodenforschung in den Sozialwissenschaften gilt es daher weiter voranzutreiben, um allen zukünftigen gesellschaftlichen Herausforderungen begegnen und Chancen für die empirischen Sozialwissenschaften nutzen zu können.

Für das Schwerpunktheft zeichnet die Plattform für Umfragen, Methoden und empirische Analysen (PUMA) verantwortlich. PUMA ist ein österreichweites Kooperationsprojekt zwischen der Universität Wien (Leitung), der Johannes-Kepler-Universität Linz, der ParisLodron-Universität Salzburg, Alpe-Adria-Universität Klagenfurt, Leopold-Franzens-Universität Innsbruck, der Karl-Franzens-Universität Graz sowie der Statistik Austria. Assoziierte Partnerorganisationen sind die Österreichische Akademie der Wissenschaften und die MODUL University. Finanziert aus den Hochschulraumstrukturmitteln 2013 durch das Bundesministerium für Bildung, Wissenschaft und Forschung (BMBWF) hat PUMA zwischen 2014 und 2018 zahlreiche öffentlich ausgeschriebene Datenerhebungen zu zentralen gesellschaftlich relevanten und innovativen Fragestellungen durchgeführt, methodische Weiterbildungen und Austauschworkshops veranstaltet und öffentliche Symposien zur sozialwissenschaftlichen Umfrageforschung in Österreich organsiert.

Das vorliegende Schwerpunktheft wurde von Sylvia Kritzinger und Katharina Götsch gestaltet und betreut.

Unser Dank gilt dem Redaktionsteam der Österreichischen Zeitschrift für Politikwissenschaft für die Möglichkeit, in einem Schwerpunktheft aktuelle Themen und Herausforderungen für Qualitätsstandards der quantitativen Sozialwissenschaften zu diskutieren, sowie für die ausgezeichnete Begleitung und Unterstützung in diesem Prozess. Insbesondere gilt unser Dank dem ÖZP-Managing Editor Dr. Mario Wintersteiger für seine hervorragende Arbeit.

Den GutachterInnen danken wir für zahlreiche konstruktive Hinweise und eine kritische Analyse der Forschungsartikel.

Nicht zuletzt möchten wir uns bei den Autorinnen und Autoren für ihre Beiträge und enge Kooperation bedanken, die dieses Schwerpunktheft erst möglich gemacht haben.

\section{Autoren}

Sylvia Kritzinger ist Professorin für Methoden in den Sozialwissenschaften am Institut für Staatswissenschaft der Universität Wien. Sie ist die Leiterin des PUMA-Projekts, Co-Projektleiterin der Austrian National Election Study (AUTNES) und spezialisiert auf Umfragedaten. Sie forscht zu Themen der öffentlichen Meinung und des Wahlverhaltens.

Katharina Götsch ist Politikwissenschafterin und seit 2014 am Institut für Staatswissenschaft der Universität Wien für die Koordination der PUMA-Plattform verantwortlich. Darüber hinaus ist sie als Lehrbeauftragte an verschiedenen Universitäten und Fachhochschulen tätig. 
